\title{
Linx
}

Revue des linguistes de l'université Paris X Nanterre

$80 \mid 2020$

L'héritage de Jean Dubois et Françoise Dubois-Charlier

\section{La causation en français : la construction transitive et la construction en FAIRE}

Causation in French: the transitive construction and the FAIRE construction

\section{Geneviève Girard-Gillet}

\section{(2) OpenEdition}

\section{Journals}

Édition électronique

URL : http://journals.openedition.org/linx/6263

ISSN : 2118-9692

\section{Éditeur}

Presses universitaires de Paris Nanterre

\section{Référence électronique}

Geneviève Girard-Gillet, « La causation en français : la construction transitive et la construction en FAIRE », Linx [En ligne], 80 | 2020, mis en ligne le 10 juillet 2020, consulté le 05 août 2020. URL : http:// journals.openedition.org/linx/6263; DOI : https://doi.org/10.4000/linx.6263

Ce document a été généré automatiquement le 5 août 2020.

Département de Sciences du langage, Université Paris Ouest 


\title{
La causation en français : la construction transitive et la construction en FAIRE ${ }^{1}$
}

\author{
Causation in French : the transitive construction and the FAIRE construction
}

\author{
Geneviève Girard-Gillet
}

1 Le français possède différents outils pour exprimer linguistiquement la causation, mais nous ne nous intéresserons ici qu'à deux moyens, l'utilisation de verbes transitifs qui expriment l'action d'un agent sur un patient et le recours à la construction FAIRE faire. L'utilisation de verbes transitifs pose déjà un problème délicat, car la possibilité de transformer le vent casse la branche, où le causateur est le sujet du changement d'état, en la branche casse, où le patient devient sujet, n'est possible que pour un nombre assez limité de verbes, les verbes dits « symétriques " (Dubois : 1967). Travaillant à partir de l'ouvrage de Jean Dubois et Françoise Dubois-Charlier, Les Verbes français (1997), Imen Ben Salah Tlili (2007) en donne la liste : il y en a environ 300 (2007). En l'absence d'un fonctionnement " ergatif $»^{2}$ généralisable à tous les verbes, fonctionnement qui existe en anglais et en espagnol, le français a recours à la construction en FAIRE : Marie a fait voler son cerf-volant. L'existence de cette construction pose alors la question du choix entre : Jean a cuit le lapin / Paul a fait cuire le lapin, cas où les deux structurations sont possibles ${ }^{3}$.

2 Pour rendre hommage à Jean Dubois et Françoise Dubois-Charlier, nous évoquerons leurs travaux sur les verbes du français, et montrerons en quoi leur l'hypothèse sur l'« adéquation entre les schèmes syntaxiques de la langue et l'interprétation sémantique ${ }^{4}$ " est particulièrement pertinente pour notre problématique, et pourquoi la langue peut, de ce fait, adopter de nouvelles structurations.

3 Notre article comprend trois parties :

4 - discussion des deux types de causation : causation externe / causation interne.

5 - études des verbes symétriques qui expriment la causation. 
6 - hypothèse sur l'acceptabilité de nouvelles constructions causatives telles que la suivante : ils l'ont suicidé.

7 Cet article reprend et précise des hypothèses posées précédemment.

\section{La causation}

\subsection{Deux types de causation}

8 Jacqueline Guéron (2008) fait l'hypothèse qu'il faut poser une distinction entre deux types de causation, la causation qu'elle appelle intentionnelle et la causation qu'elle appelle inertielle. Dans la causation intentionnelle, la cause de l'événement est l'intention d'un causateur humain, alors que dans la causation inertielle, il n'y a pas d'action d'un acteur humain, mais ce sont les lois de la nature qui règlent les changements d'état qui se mettent en place au cours du temps. Levin (2009) fait le même type de distinction et considère que la causation externe est l'action d'un agent pour obtenir un résultat :

causation externe : result (i.e., externally caused) state $\rightarrow[[\mathrm{x}$ ACT $]$ CAUSE [BECOME

[y $<$ RES-STATE> ] ]]

C'est ce qui est exprimé par des verbes tels que casser / sécher/ fondre/ ouvrir ..., alors que la causation interne ne nécessite pas l'action d'un agent :

causation interne : internally caused state $\rightarrow[\mathrm{x}<$ state $>]$.

Les événements arrivent inexorablement, dans l'ordre naturel des choses, en fonction des lois de la nature. C'est ce que disent les verbes fleurir, bourgeonner, faner, rouiller, moisir ...

11 Le point important est que Levin pose une corrélation entre la causation externe et la transitivité, et entre la causation interne et la non transitivité. La syntaxe permet ainsi l'expression de différences sémantiques. C'est l'hypothèse des Dubois : il y a adéquation entre les schèmes syntaxiques de la langue et l'interprétation sémantique qu'en font les locuteurs.

12 Talmy (1976, 1985 : 78-85) avait déjà suggéré qu'il y avait une interaction entre l'agoniste, celui qui agit, et l'antagoniste, celui sur lequel il agit ; et en d'autres termes, qu'il fallait creuser les données sémantiques à l'œuvre. Et Croft (1992:173), quant à lui, parlait de relations asymétriques entre l'instigateur du changement et le lieu du résultat du changement, et donc de la relation agent/patient-thème.

13 Si l'hypothèse de Levin sur la bi-univocité du lien syntaxe/interprétation n'est pas confirmée dans tous les cas de figure, comme le montrent McKoon et Macfarland (2002), avec la possibilité d'avoir the intense heat wilted the flowers, the severe frost withered the tulips, ${ }^{5}$ il n'en reste pas moins que le lien entre syntaxe et sémantique est un lien fort, ce que nous allons essayer de montrer à partir du fonctionnement des verbes causatifs.

\subsection{Quelques exemples de l'opposition entre les deux types de causation.}

Lorsque je dis : Jean a fait bouillir de l'eau, l'événement exprimé par cet énoncé se décompose en plusieurs sous-événements : Jean a mis de l'eau dans la casserole, a 
allumé le feu, puis l'eau s'est mise à chauffer puis elle est arrivée à ébullition. La causation externe est l'action de Jean sur la casserole, qui va se mettre à chauffer, puis à bouillir, et la causation interne est le fait que dans un environnement chaud, l'eau ne peut que se mettre à bouillir. En d'autres termes, Jean peut quitter la pièce sans intervenir davantage ; une fois le processus de chauffage enclenché, l'eau va petit à petit chauffer puis bouillir ${ }^{6}$. Elle le fait du fait de ses caractéristiques intrinsèques, internes, de sa propension à chauffer. Si Jean avait mis une pierre dans la casserole, elle ne se serait pas mise à bouillir dans les mêmes circonstances.

La causation externe c'est Jean + la chaleur. Jean initie le début de la chaleur, et ensuite la chaleur devient l'agent causateur du changement d'état. C'est peut-être par anthropomorphisme que l'environnement « chaleur » n'est qu'indirectement présent linguistiquement dans le verbe bouillir, et que Jean semble être le seul responsable du changement d'état. Doit-on d'ailleurs décomposer l'énoncé en :

(1) Jean a fait [l'eau-bouillir] ou en :

(2) Jean a fait [la chaleur a fait [l'eau-bouillir] $]^{7}$

La deuxième décomposition permet de poser toutes les entités à l'œuvre pour atteindre le résultat, à savoir que l'eau bout : l'action de Jean, la chaleur, et la transformation de $l^{\prime}$ entité, eau, ${ }^{8}$ quand la température atteint $100^{\circ}$. La distinction entre l'action causatrice de Jean -causation externe- et le processus qui se développe -causation interne- peut être montrée par un exemple tel que : Fais vite bouillir de l'eau pour le thé, où l'adverbe, vite, ne peut renvoyer qu'à la demande d'agir vite, d'ouvrir le gaz, et non au temps que mettra l'eau pour bouillir. ${ }^{9}$

a causation interne est assimilable aux caractéristiques de l'eau. Comme nous le disions plus haut, l'eau possède une caractéristique intrinsèque, à savoir sa propension à chauffer dans un certain environnement. Mais il n'est pas suffisant de bien faire la part des choses entre les deux types de causation; il faut se demander s'il y a un lien possible ou non entre les deux types. La réponse est oui ; et nous appelons ce lien l'« adéquation causale " (adequacy parameter). Cette adéquation signifie qu'un changement d'état initié par une entité A sur l'entité B ne peut être opérationnel que si $A$ possède, au moment de son intervention, les caractéristiques adéquates, les potentialités adéquates pour modifier dans $B$ les caractéristiques qui y répondent. En d'autres termes, si A chauffe, il faut que B soit sensible à la chaleur; si A mouille, il faut que B soit sensible à l'humidité. Sans cette adéquation causale, rien ne se passerait.

Cette notion d'adéquation semble être à l'œuvre dans d'autres domaines sémantiques, et elle permettrait même l'économie linguistique. La différence entre MAKE et DO en anglais (Girard 1993, 2003), est une différence sémantique forte entre le verbe MAKE, qui exprime la création d'une nouvelle entité, et le verbe DO, verbe très pauvre du point de vue sémantique, mais dont le sens se déduit de la relation entre ce que l'on sait du sujet et de ce que l'on sait de l'objet, dans un rapport d'adéquation. Voici deux exemples qui illustrent l'interprétation qu'il convient de donner à DO :

(3) "There's a lot of work waiting for you. I promised Lady Anchorage you would do

Anchorage House as soon as you got back." ${ }^{10}$ (E.Waugh, Brideshead Revisited).

Pour interpréter l'énoncé, il faut, en effet, savoir que Charles, le you de l'énoncé, est spécialiste en rénovation de vieilles demeures, et que sa femme lui propose ici de rénover une maison. Que fait un spécialiste en rénovation par rapport à une maison qui 
a besoin d'être rénovée ? Il la rénove. Sa profession est en adéquation avec ce qu'on lui demande de faire.

Voici un autre exemple :

(4) "I need a killer"

"We do not do politicians nor businessmen."11

(Kaurismaki, film J'ai engagé un tueur) tueur à qui il s'adresse lui répond que son « agence » ne tue pas les hommes politiques. L'adéquation supposée entre la spécialité du tueur et le contrat proposé se révèle fausse : le tueur ne tue pas les politiciens.

\section{Verbes « symétriques » et causation}

\subsection{Comparaison d'exemples}

Beaucoup de travaux antérieurs ont bien analysé la complexité du phénomène de symétrie, en particulier J.P. Boons, A. Guillet \& C.L. Leclère (1976), A. Zribi-Hertz (1987), R. Forest (1988), J. Dubois et F. Dubois-Charlier (1997), I. Ben Salah Tlili (2007), R. Pasero, P. Sabatier et M.-H. Stefanini (2010), et ils ont montré que la symétrie n'existe pas partout, d'où le recours à d'autres appellations, comme celle de " verbes neutres » chez J.P. Boons, A. Guillet \& C. Leclère. En dépit du doute exprimé par Lazard (1994) quant à la possibilité de définir la classe sémantique à laquelle ils appartiendraient tous, les spécialistes du domaine ont essayé de mieux définir leurs traits spécifiques. Dans son article de 2007, Imen Ben Salah Tlili discute de la possibilité de définir des catégories sémantiques en fonction du sémantisme du verbe et du sémantisme du sujet pour les emplois intransitifs. Dans la mesure où nous analysons des constructions transitives il est utile de réfléchir au sémantisme de l'objet et au lien entre le sujet et l'objet. Le recours aux deux types de causation, avec, de surcroît, des comparaisons avec la construction en FAIRE devrait nous permettre de bien saisir les diverses interprétations.

Voyons, à partir de deux exemples, en quoi la distinction entre les deux types de causation posées plus haut permet de bien comprendre comment la syntaxe se met au service du sens à construire.

(5) tu peux tremper ton croissant dans le café (Causation Externe + Causation Interne très faible) ${ }^{12}$

(6) il faut faire tremper les raisins pendant une heure (Causation Externe +

Causation Interne)

Dans ces deux premiers exemples, le même verbe, tremper, est utilisé, en (5) transitivement, et en (6) avec FAIRE. La traduction en anglais par dip pour le premier cas et soak pour le deuxième nous indique une différence conceptuelle. Quelle est-elle ? En (5) le croissant va rester une à deux secondes dans le café et il n'aura pas le temps de permettre à sa causation interne, qui est de changer de consistance dans un liquide, de se développer entièrement ${ }^{13}$; alors qu'en (6) les raisins restent longtemps dans l'eau sucrée pour qu'ils aient le temps de se modifier. Le complément de temps, une heure, renvoie au temps de trempage et non à l'action de l'agent de plonger les raisins dans le liquide. L'énoncé décompose implicitement la causation en causation externe et causation interne ${ }^{14}$. 
Considérons les exemples suivants :

(7) Voici une technique facile pour ouvrir des huîtres creuses sans se blesser (Causation Externe seule)

(8) Grattez et lavez les moules. Faites-les ouvrir sur feu vif. (Causation Externe + Causation Interne)

ouvrir les huîtres, le cuisinier se sert d'un couteau à huîtres, et les huîtres ne jouent aucun rôle dans le processus ; il n'y a pas déclenchement d'une quelconque causation interne. Si on laisse le couteau à côté des huîtres, elles ne vont pas s'ouvrir toutes seules ${ }^{15}$ (voir Fillmore (1968) et son étude du verbe cut: John cut the bread / *the bread cut). En revanche, l'ouverture des moules se fait dans une cocotte chaude, et, sous l'effet de la chaleur, les moules réagissent en s'ouvrant - causation interne. L'anglais décrit là aussi différemment les deux procès : open the oysters / steam the mussels open.

En cherchant des occurrences du verbe friser sur internet, nous trouvons des utilisations transitives et des utilisations avec FAIRE :

(9) vous avez été nombreuses à me demander comment je frise mes cheveux

(10) l'air marin avait fait friser mes cheveux

(11) j'ai essayé la laque et ça fait friser les cheveux

(12) pourquoi la pluie fait friser les cheveux ?

Il apparaît, à partir de ces quelques exemples ${ }^{16}$ que l'énonciateur fait une distinction entre un énoncé qui décrit qu'un agent cause le changement d'état des cheveux -le premier- et un énoncé qui exprime le moyen par lequel le changement d'état a eu lieu : la laque, par exemple, ou bien encore un énoncé qui traduit l'action de certaines lois de la nature : l'air marin, la pluie.

31 Comment pouvons-nous expliquer le choix entre les deux constructions?

\subsection{Hypothèse sur les types d'agent}

Les travaux sur les verbes symétriques, et ceux de J. Dubois et F. Dubois-Charlier, entre autres, ont montré que les verbes qui sont toujours symétriques sont souvent des verbes appartenant au domaine culinaire.

(13) le cuisinier mijote le ragoût $\rightarrow$ le ragoût mijote.

(14) le cuisinier marine les harengs $\rightarrow$ les harengs marinent. ${ }^{17}$

Cela est exact, mais les recherches menées depuis 2015 avec Claude Delmas sur les recettes de cuisine en français tendent à montrer que la construction en FAIRE apparaît de manière systématique dans les conseils donnés au cuisinier, faites mijoter le ragoût une heure, faire frire les oignons, faire cuire à feu doux, etc. Il faut donc se demander pourquoi le français opte pour la construction en FAIRE.

Les exemples proposés par J. Dubois et F. Dubois-Charlier ont toujours pour sujet un spécialiste du domaine, à savoir ici, des cuisiniers, pour les verbes appartenant au domaine de la cuisine. Mais nous pouvons faire la même remarque pour les exemples avec les coiffeurs, les médecins :

(15) le coiffeur frise mes cheveux

(16) le médecin a accouché ma sœur activités dont ils sont spécialistes. Nous retrouvons ici la notion d'adéquation causale 
évoquée plus haut. Internet nous fournit de nombreuses occurrences, qui vont nous permettre de préciser les tendances.

(17) le menuisier vieillit les meubles pour les vendre comme des antiquités

(18) les vignerons vieillissent le vin dans des fûts de chêne.

Dans tous ces exemples, il y a focalisation sur l'agent humain de la modification. Les énoncés ne nous disent pas, en effet, les moyens utilisés par les agents, car c'est leur spécialité qui est posée comme garante à elle seule de la bonne marche des opérations. L'énonciateur s'intéresse à l'agent humain uniquement.

Mais si l'agent n'est pas un spécialiste du domaine, la construction avec FAIRE semble préférable.

(19) Voici les conditions de bonne conservation pour ceux qui font vieillir nos vins $\mathrm{d}$ 'après les conseils des vignerons d'Anjou. (books.google.fr>books, J.Bellard, Vineyards, 1998)

(20) La cave à vin électrique peut alors être une bonne option pour faire vieillir votre vin selon vos besoins. (https://caves-aubonplaisir.fr/comment-conserver-vin)

En revanche, lorsque c'est l'agent naturel, l'environnement, ${ }^{18}$ qui est mis en avant, la construction transitive semble préférée :

(21) aucune lumière naturelle n'est admise dans une cave à vin, car elle vieillit le vin trop rapidement. (http:// www.emballage-bouteille-vin.fr/7-regles-a-respecter) (22) La chaleur a flétri le bouquet de freezia; il n'en reste que la fragrance. (https:// lombradelleparole.wordpress.com/tag/lao-tzu/)

(23) le sel cuira la chair de votre saumon

(24) il ne faut surtout pas arroser pendant la journée lorsque les plantes sont en plein soleil car la réverbération risque de les brûler. (https:// boutdejardin.lebonforum.com/t16-les-principes-d-un-bon-arrosage)

Dans tous ces énoncés, ce n'est pas l'agent humain qui intéresse l'énonciateur. Ce dernier peut seulement faire en sorte que de bonnes conditions existent pour que le résultat souhaité soit atteint :

- il peut éviter qu'il y ait de la lumière dans la cave, mais c'est la lumière qui a une influence. - il peut ajouter du sel dans la préparation du saumon, mais c'est le sel qui agira.

il peut éviter d'arroser, mais c'est la réverbération du soleil qui est dangereuse pour les plantes. On a pour les mêmes raisons :

(25) la neige a brûlé l'herbe.

Dans la mesure où il est difficile d'exclure complétement l'utilisation d'une structure, nous suggérons que c'est à l'énonciateur de faire le choix qui correspond au message qu'il veut faire passer ; il peut privilégier l'action de l'agent humain, si celui-ci peut effectivement agir ${ }^{19}$, ou au contraire l'agent " naturel ». La construction avec FAIRE tend à suggérer indirectement qu'un « causateur » intermédiaire vient s'interposer au cours d'un certain procès.

Dans les cas où c'est un humain qui est l'agent, deux constructions peuvent s'opposer :

(26) Marie a brûlé la lettre

(27) Marie a fait brûler la lettre

Dans le premier cas, l'énoncé signifie, d'après nous, que Marie a volontairement détruit la lettre, parce que son contenu la blessait, par exemple. La construction exprime 
l'intentionnalité de Marie, et c'est Marie, et elle seule, qui a obtenu le résultat souhaité. Mais en (27), nous comprenons que c'est un accident, suite à un moment d'inattention peut-être. Marie faisait réchauffer un plat, tout en lisant une lettre, et celle-ci a pris feu. On aura de même, Paul a fait brûler la casserole en oubliant d'éteindre le gaz, plutôt que ? Paul a brûlé la casserole, ${ }^{20}$ dans le contexte de la préparation d'un repas. Ces structures posent, en fait, une opposition entre un fond de casserole qui a brûlé, et une casserole qui a été entièrement détruite volontairement. Divers paramètres de nature pragmatique doivent donc être pris en considération pour construire l'interprétation.

\subsection{Limites des emplois symétriques ${ }^{21}$}

Nous venons de voir que les agents humains peuvent être à l'origine de certains changements d'état, mais ils ont besoin, dans de nombreux cas, d'agents naturels dont les pouvoirs modificateurs sont en adéquation avec les potentialités des entités considérées. Que se passe-t-il si les entités ne peuvent pas réagir aux modifications exercées sur elles? Si nous comparons :

(28) les soucis ont vieilli Marie prématurément

(29) ce chapeau vieillit Anne

nous comprenons que Marie a vieilli, mais qu'Anne, elle, n'a pas vieilli. Pourquoi ? Tout être humain a une propension inhérente au vieillissement, et si des circonstances négatives, comme des soucis, interviennent, alors cette propension au vieillissement s'actualise ou s'aggrave. En revanche, le chapeau ne possède pas de virtualités capables d'entrer en relation avec la propension au vieillissement que tout être humain possède. Il ne peut donc pas faire vieillir quelqu'un. On a une interprétation subjective : c'est l'énonciateur qui trouve que la personne paraît plus vieille avec ce chapeau.

Pour Marie, les soucis sont la causation externe à l'actualisation de son vieillissement, qui relève de la causation interne. Pour Anne, il y a bien une causation externe, le port $\mathrm{du}$ chapeau, mais cette causation n'entre pas en résonnance avec un vieillissement possible.

(30) il a mijoté le ragoût pendant une heure $\rightarrow$ le ragoût a mijoté pendant une heure (he simmered the stew for one hour / the stew simmered for one hour) ${ }^{22}$

Il est intéressant de noter que dans les recettes on trouve plutôt laisser que faire : laisser mijoter un quart d'heure, ce qui confirme l'idée que le ragoût continue à mijoter de par ses caractéristiques internes, c'est-à-dire sans l'action du cuisinier (absence de causation externe), ce que le sémantisme de laisser suggère. ${ }^{23}$

3 En (31) c'est un sens métaphorique qui est construit :

(31) il mijote un mauvais coup (he's cooking up some mischief/ he's up to some mischief)

et il semble difficile d'en déduire : ? un mauvais coup mijote.

(32) si tu arroses trop tes salades, tu vas les faire pourrir $\rightarrow$ elles vont pourrir ${ }^{24}$

(33) le tabac a pourri la vie de mon frère $\rightarrow$ ?* sa vie a pourri

(34) l'humidité ramollit les biscuits $\rightarrow$ ils ramollissent ${ }^{25}$

(35) trop de publicité ramollit le cerveau $\rightarrow$ le cerveau se ramollit (sens métaphorique)

(36) la lumière a vieilli ton vin $\rightarrow$ le vin a vieilli

(37) sa barbe le vieillit $\rightarrow$ il n'a pas vieilli (c'est juste une impression) 
(38) le vent remue les feuilles $\rightarrow$ les feuilles remuent

(39) ce texte a remué Marie $\rightarrow$ * Marie a remué

Les emplois métaphoriques ne permettent pas au verbe de fonctionner de manière symétrique. Cela s'explique, nous l'avons vu, par le fait que la causation interne de l'entité sur laquelle agit le causateur, qu'il soit humain ou non, et qui aurait dû réagir par adéquation causale n'existe pas. Sans adéquation causale, le causateur ne peut exercer aucun pouvoir sur l'entité en question. En (37), la barbe n'a pas la capacité (causation externe) d'ajouter des ans à l'homme qui la porte, elle ne peut donc pas faire en sorte que l'homme vieillisse, bien que l'homme possède une propension au vieillissement. En revanche en (36), la lumière, dont on sait qu'elle est un facteur de vieillissement, peut agir sur le vin, qui possède une certaine propension au vieillissement.

Nous allons étudier maintenant en quoi cette absence d'adéquation causale nous permet de comprendre les évolutions actuelles de la grille argumentale de certains verbes, qui, d'un fonctionnement intransitif, passe à un fonctionnement transitif.

\section{Hypothèse sur l'acceptabilité de nouvelles constructions causatives}

L'existence d'utilisations transitives de verbes intransitifs pose alors la question de leur intégration dans le système linguistique du français, car les nouvelles structures ne doivent pas générer des ambigüités interprétatives. Nous avons remarqué que ces constructions existent avec les trois verbes intransitifs suivants, suicider, exploser, démissionner, mais il peut exister d'autres verbes qui fonctionnent de la même manière. Notre recherche porte exclusivement sur ces trois verbes, dont nous avons cherché des exemples sur Internet :

(40) Je ne le connais que de nom. C'est bien lui qui s'est suicidé, n'est-ce pas?

Ils l'ont suicidé ! Jérôme se rapprochait trop de la cause cathare [...] Ils l'ont tué ; ce sera bientôt mon tour. (Daniel Hernandez, le Loup des Cathares, 2017)

(41) Mozart a découvert le pot aux roses, poursuivit la princesse, et ils l'ont suicidé (Oscar Peterson, L'imposture nucléaire, 2017)

(42) Il voit un homme dans les airs, en chute libre. Impossible de dire si on l'a poussé, ou s'il a sauté. "Ils l'ont suicidé » résumera-t-on plus tard en une phrase qui deviendra tristement célèbre. (Paolo Roversi, La ville rouge, 2012)

(43) Ce scandale! Ils l'ont suicidé pour pas qu'il parle. Epstein avait des dossiers sexuels sur tous les puissants. (10 août 2019, https://twitter.com/marteauolivier/status) (44) En gros, le FELV ça détruit les globules rouges, ça les explose. Elle a 2 chances sur 3 de mourir. (https://twitter.com/Spinochat/status/890540889201926144)

(45) L'incroyable Usain Bolt a encore frappé. Il a explosé le record du monde du 200 m. (https://www.purepeople.com/article/l-incroyable-usain-bolt-a-encore-frappe-il-aexplose-le-record-du-monde-du-200m-regardez_a38214/1)

(46) Parasidose, les poux, les lentes, ça les explose (https://www.youtube.com/watch? $v=w L f Z d 2 l 3 l i o$ )

(47) Ils l'ont démissionné ! Il est remplacé par José Merlot ! Au fond de lui-même, Boris savait que pareille catastrophe allait se produire d'un jour à l'autre. (Maryse Condé, La belle créole, 2003)

(48) C'est bien de refuser de partir (pourquoi devrait elle ?) mais de toutes façons ils vont la démissionner, comme les précédents. (www.thailande-fr.com/actu/29666yingluck-shinawatra-reste-attachee-fauteuil) 
58 Ces constructions peuvent donc entrer dans la syntaxe du français, sans qu'elles génèrent de l'ambigüité. Sans cette distinction de nature cognitive, ces nouvelles constructions transitives n'auraient peut-être pas pu voir le jour. L'énonciateur les adopte pour mettre l'accent sur le caractère non attendu du changement d'état, car celui-ci doit frapper les esprits. Elles sont l'expression d'un excès, d'un haut degré, voire d'une polémique quand le décès d'un individu est source d'interrogation, et participent, de fait, de la mirativité.

\section{Conclusion}

Le français, nous venons de le voir, a la possibilité de structurer différemment les divers types de causation ${ }^{26}$ dont l'énonciateur est le témoin, en particulier lorsque ce dernier souhaite focaliser l'attention du récepteur sur la causation externe ou sur la 
causation interne du processus. Cela ne signifie pas que cette possibilité existe dans tous les cas de figure. L'énonciateur a parfois le choix, mais dans d'autres contextes, il est plus contraint. La pragmatique a son rôle à jouer, mais une distinction semble toujours présente, entre l'agent humain qui manipule les entités et les agents naturels existant dans l'environnement, et qui sont, en dernière analyse, les véritables responsables de certains changements d'état. Si l'on peut dire la neige a brûlé l'herbe, il est plus difficile de dire, en constatant que la recette est ratée, ? Jean a brûlé les oignons. On préférera Jean a fait brûler les oignons, ce qui sous-entend que l'action de Jean sur les oignons - il a fait quelque chose - s'est trouvée affectée par une autre donnée, la chaleur du gaz, parce qu'il la maîtrisait mal. Le résultat, une poêle pleine de charbon, est la conséquence de l'action de Jean + l'action d'une chaleur trop forte, ce qui a contrecarré le résultat souhaité.

De cette étude rapide, il ressort que de nombreux paramètres interviennent dans l'interprétation des énoncés, et que la compréhension de la relation syntaxesémantique nécessite toujours plus de réflexion, en particulier pour essayer de rendre compte de certaines structures émergentes.

\section{BIBLIOGRAPHIE}

Abeillé, A., Godard, D., Miller, P. (1997). « Les causatives en français, un cas de compétition syntaxique ", in Langue française, $\mathrm{n}^{\circ} 115$, pp. 62-74. Paris : Larousse.

Ben Salah Tlili, I. (2007). « Contribution à l'étude des « verbes symétriques » en français contemporain », in Cahiers du CRISCO, $\mathrm{n}^{\circ} 23, \mathrm{pp} .16-37$.

Alexiadou, A., Anagnostopoulou, E. (2003). "Voice morphology in the causative-inchoative alternation: Evidence for a non-unified structural analysis of unaccusatives", in A. Alexiadou, E. Anagnostopoulou, \& M. Everaert (éds.), The unaccusative puzzle: Explorations of the syntax-lexicon interface, pp. 114-136. Oxford: Oxford University Press.

Alexiadou, A., Anagnostopoulou, E., Schäfer, F. (2006). "The properties of anti-causatives crosslinguistically", in M. Frascarelli (éd), Phases of interpretation, pp.187-211. Berlin: Mouton de Gruyter. doi: 10.1515/9783110197723.4.187

Boons, J.P., Guillet, A., Leclère, C. (1976). La structure des phrases simples du français : constructions intransitives. Genève : Droz.

Chatti, S. (2009). Sémantique de la causation analytique. PhD Dissertation, Université Sorbonne Nouvelle Paris 3.

Collins, J., Hall, N., Paul, L.A. (éds) (2004). Causation and Counterfactuals. Cambridge, Mass.: The MIT Press.

Cotte, P. (1993). « Ces mouvements qui font signe », in Faits de langues, $\mathrm{n}^{\circ} 1, \mathrm{pp} .129-136$. Paris : PUF. doi: 10.3406/flang.1993.1044

Croft, W. (1994). "The semantics of subjecthood", in M. Yaguello (éd), Subjecthood and Subjectivity, the status of the subject in linguistic theory. pp. 29-75. Paris : Ophrys. 
DeLancey, S. (1984). "Notes on agentivity and causation", in Studies in Language, n ${ }^{\circ}$, pp. 181-213. doi: 10.1075/sl.8.2.05del

Delechelle, G. (1989). L'expression de la cause en anglais contemporain. PhD Dissertation, Université Sorbonne Nouvelle Paris 3.

Delmas, C. (2001). « La sémantique propose, la syntaxe dispose », in QWERTY, n 11, pp. 247-255. Pau : P.U.Pau.

Delmas, C. (2004). « Fragments d'un discours culinaire », in L. Gournay \& J.-M. Merle (éds), Contrast, Mélanges offerts à J. Guillemin-Flescher, pp. 25-34. Paris : Ophrys.

Delmas, C., Girard-Gillet, G. (2019). « Brièveté et contrainte pragmatique dans le discours culinaire », in Faits de Langues, n 49, 2, pp. 69-80. Leiden/Leyde : Brill (brill.com/abstract/ journals/fdl/49/2/fdl.49.issue-2.xml)

Dixon, R.M.W., Anderson, S.R. (1994). Ergativity. Cambridge: Cambridge University Press.

Dubois, J., Dubois-Charlier, F. (1997). Les Verbes français. Paris : Larousse.

Dubois, J., Dubois-Charlier, F. (2011). Les Verbes français. rali.iro.umontreal.ca/Dubois.

Fillmore, C. (1968). "The case for Case", in Bach, Emmon \& Harns (éds), Universals in Linguistic Theory, pp. 1-88. New York: Holt, Rinehart \& Winston.

Forest, R. (1988). « Sémantisme entéléchique et affinité descriptive : pour une ré-analyse des verbes symétriques ou neutres en français ", in Bulletin de la société linguistique de Paris, $n^{\circ} 83$ (1), pp. 137-162.

François, J., Rauh, G., Broschart, J. (1994). « Les relations actancielles, sémantique, syntaxe, morphologie », in Langages, n² 28, pp. 113-127. doi: 10.3406/lgge.1994.1667.

François, J., Le Pesant, D., Leeman, D. (2007). « Présentation de la classification des Verbes Français de Jean Dubois et Françoise Dubois-Charlier », in Langue française, ${ }^{\circ}$ 53. pp. 3-19.

Girard-Gillet, G. (1999). « Make/Lassen/Faire, Semantic delimitation and syntactic construction, in Contrast, Comparison and Communication », in CIEREC, Travaux XCVI. pp. 49-60. St Etienne.

Girard-Gillet, G. (2003). « L'interprétation de do lexical », Journée ALAES, https://

alaesfrance.files.wordpress.com/2015/04/girard2003.pdf

Girard-Gillet, G. (2015). La quantification des temporalités dans les recettes de cuisine en anglais », in D. Banks (éd), La quantification dans le texte de spécialité, pp. 141-157. Paris :

l'Harmattan.

Girard-Gillet, G. (2016). « Atypical argument structures in French: From metaphorical uses to atypical ones », in T. Ruchot \& P. Van Praet (éds) Atypical predicate-argument relations, pp. 181-202. Amsterdam: John Benjamins.

Girard-Gillet, G., Delmas, C. (2019). « Remarques sur la brièveté syntaxique dans les recettes de cuisine anglaises », in I. Behr \& F. Lefeuvre (éd), Le genre bref, pp. 131-146. Berlin : Frank \& Timme. Guéron, J. (2008). « Visible effects, hidden causes », in G. Girard-Gillet (éd), L'envers du décor, Etudes de linguistique anglaise, pp. 158-179. Université d'Avignon.

Haspelmath, M. (1993). "More on typology of inchoative/causative verb alternations", in B. Comry \& M. Polinsky (éds), Causatives and transitivity, pp. 87-120. Amsterdam: John Benjamins.

Holmes, J. (1999). "The Syntax and Semantics of causative verbs", in UCPWPL, n 11, pp. 323-348. 
Koenig, J.-P., Mauner, G., Bienvenue, B., Conklin, K. (2007). What with?: The anatomy of a (proto)-role. Ms. University of Buffalo.

Langacker, R. W. (1987). Foundations of cognitive grammar, vol. 1. Stanford: Stanford U.P.

Larjavaaara, M. (2016). « The semantic motivation of non-canonical predicative relations, the French transitive construction ", in Lingvisticae Investigationes Supplementa, vol 33. pp.163-179. John Benjamins.

Lazard, G. (1994). L'Actance. Paris : Presses Universitaires de France.

Leeman, D., Sabatier, P. éds. (2011). Empirie, Théorie, Exploitation : le travail de Jean Dubois sur les verbes français, Langages nº 179-180. Paris : Larousse.

Lemmens, M. (1998). Lexical perspectives on transitiviy and ergativity: Causative constructions in English. Amsterdam: John Benjamins. doi: 10.1075/cilt.166

Levin, B. (1993). English verb classes and alternations. A preliminary investigation. Chicago and London: The University of Chicago Press.

Levin, B. (2009). "Further explorations of the landscape of causation: comments on Alexiadou and Anagnostopoulou", in MIT working papers, pp. 239-266, Cambridge: MIT.

McKoon, G., Macfarland, T. (2002). "External and internally caused change of state verbs," in Language, $\mathrm{n}^{\circ} 76, \mathrm{pp} .833-856$.

Pasero, R., Sabatier, P. Stefanini, M.-H. (2010). « Syntaxe et sémantique formelle des constructions verbales exprimant des relations symétriques ", in D. Leeman \& P. Sabatier (éds)

Pinker, S. (2008). The stuff of thought. London: Penguin.

Talmy, L. (2000). Toward a cognitive semantics, vol. 2, Cambridge, Mass: MIT Press.

Tesnière, L. (éd) (1958). Eléments de syntaxe structurale. Paris : Klincksieck. 5th edition, 1988.

Van Valin R. D., LaPolla, R. J. (1997). Structure, meaning and function. Cambridge: CUP. doi:10.1017/ CBO9781139166799.

Zribi-Hertz, A. (1987). « La réflexivité ergative en français moderne », in Le Français moderne, $\mathrm{n}^{\circ} 55$, pp. 23-54.

\section{NOTES}

1. Nous remercions deux relecteurs dont les remarques très pertinentes nous ont permis, nous l'espérons, d'améliorer notre analyse. Nous sommes responsable de toute maladresse ou erreur subsistantes.

2. Le terme « ergatif » employé ici ne renvoie pas au fonctionnement des langues dites ergatives, dans le sens de Dixon (1994), mais simplement au retournement des arguments, avec l'objet/ patient qui devient sujet. L'anglais fonctionne de manière «ergative » et peut dire : I bounced the ball (j'ai fait rebondir la balle), alors que le français ne peut pas dire : *j'ai rebondi la balle. A noter que l'espagnol fonctionne aussi de manière ergative, herví por 30 minutos (fais bouillir $30 \mathrm{mn}$ ), cocinar en un horno (faire cuire au four).

3. Nous n'envisageons pas ici l'interprétation suivante possible Paul a fait cuire le lapin à Jean.

4. J. François, D. Le Pesant, D. Leeman, 2007, Langue française, $n^{\circ} 53$.

5. Le froid intense a fait flétrir les fleurs, les gelées sévères ont fait faner les tulipes. Il est possible également de traduire par le froid intense a flétri les fleurs, mais les gelées ont fané les tulipes semble plus délicat. 
6. Dans une émission de radio consacrée à la cuisine, un présentateur expliquait qu'une fois que l'on avait mis tous les ingrédients dans la casserole, « ils se débrouillaient tous seuls pour cuire. » 7. Un relecteur suggère la décomposition suivante : «Jean a fait [x a bouilli l'eau] » parallèlement à : «Jean a fait [x a coupé son cerisier] » pour : «Jean a fait couper son cerisier ». Nous sommes d'accord, mais notre suggestion tient compte de la nécessité, nous semble-t-il, de poser que rien ne se passerait sans le rôle de la chaleur. Pour le cerisier, la décomposition note seulement qu'à l'agent possible, Jean, est substitué un autre agent. Il n'y a pas de "causateur" intermédiaire.

8. Il n'est néanmoins pas possible de construire ?*la chaleur a bouilli l'eau. Il semble que le sémantisme de bouillir pose un « achèvement " (achievement), au sens de Vendler, et non un " accomplishment ». La notion d'“ accomplishment » suggère un processus qui mène petit à petit à un résultat. On peut dire : la chaleur a petit à petit flétri mes salades ; mais il semble plus difficile de dire ? le vent a petit à petit cassé la branche, car casser exprime un achèvement, comme bouillir. Ce point demande réflexion.

9. On peut se poser la question de la taille de la casserole, bien sûr, mais ce qui doit être vite accompli est l'action d'allumer le gaz.

10. «Il y a beaucoup de travail qui t'attend. J'ai promis à Lady Anchorage que tu t'occuperais de Anchorage House dès ton retour.»

11. "J'ai besoin d'un tueur à gage »; " Nous ne nous occupons ni des hommes politiques ni des hommes d'affaire. »

12. Les exemples sont soit des exemples inventés, mais soumis à des francophones nonlinguistes, soit des énoncés collectés dans des manuels ou émissions de cuisine au cours des recherches effectuées avec C. Delmas depuis 2015.

13. Un relecteur suggère que la causation interne est mise à contribution, puisque le croissant va se ramollir un peu. C'est tout à fait exact. Nous suggérons donc une causation interne faible, du fait du peu de temps passé dans le café. Mais pour les raisins, la cuisinière sait qu'ils doivent rester assez longtemps dans le récipient pour s'imprégner du parfum du liquide.

14. L'énoncé possible Fais vite tremper les raisins nous rappelle Fais vite bouillir de l'eau, énoncé vu plus haut, où l'accent est mis sur l'initiation du procès. Mais il nous semble difficile de construire : *? Fais vite tremper les raisins pendant une heure, dans la mesure où l'agent ne peut agir que pour initier les changements d'état, et non pour influencer la durée que va prendre le changement d'état concernant les raisins.

15. La différence entre le couteau et l'eau est que le couteau n'agit que sous le contrôle de l'agent humain qui le tient dans la main, alors que l'eau, dès que l'agent humain la met à chauffer, poursuit le processus qui a été enclenché du fait de ses propriétés internes. Cette distinction explique en partie l'impossibilité d'avoir : John cut the bread $\rightarrow{ }^{*}$ the bread cut, puisque le pain ne possède pas une capacité inhérente à se couper lui-même, quel que soit le contexte. En revanche, l'eau, dans un contexte adéquat, va se mettre à chauffer.

16. Nous n'avons pas de statistiques ; nous remarquons surtout des tendances, mais aucune structure n'est a priori exclue. L'énonciateur peut décider, pour des raisons pragmatiques, de mettre ou non au premier plan l'agent humain, si la langue le permet. Il en va différemment avec un énoncé tel que :j'ai fait friser mes cheveux par ma sœur, où l'énonciateur a recours à FAIRE pour indiquer qu'un acteur différent de lui est le causateur du changement d'aspect des cheveux. Nous ne traitons pas de ce cas de figure ici, car il demande une étude spécifique.

17. Le Trésor de la Langue Française ne donne aucun exemple transitif, nous fait remarquer un relecteur, que nous remercions.

18. Nous optons, pour l'instant, pour ces termes. Par " naturel ", " environnement " nous entendons les éléments fort variés de la nature qui conditionnent les modifications souhaitées ou non par l'agent humain. C'est la chaleur pour que l'eau puisse bouillir, c'est l'eau pour que les plantes poussent, etc. Les lois de la nature qu'évoquent Guéron (2008) et Levin (2009) sont bel et bien à l'œuvre, mais elles sont mises à profit ou non par un agent humain qui évalue ses besoins. 
19. Il n'est pas possible de dire dans le contexte du jardinage, à la différence de celui de la cuisine : *Jean a flétri les salades, *Jean a fleuri les plantes. McKoon et Macfarland (2002) donnent un exemple intéressant en anglais, à propos de la déesse Déméter : "Démeter harvested the crops each year, blossomed the flowers, grew grass and had a daughter named Persephone, ruler of the underworld". (En tant que déesse de la nature, elle commande aux plantes). Donc l'anglais permet, en traduction mot à mot : « elle fleurit les fleurs, pousse l'herbe ».

20. Pinker (2008: 81) fait remarquer que lorsqu'une langue a plusieurs structures pour exprimer la causation, elle aura tendance à utiliser la forme la plus concise pour traduire la causation directe, et la plus prolixe pour la causation indirecte. "Some languages have various ways of describing a causative event. Whenever a language contains more than one of these services it uses the more concise for direct causation and the more prolix one for less direct causation."

21. Nous travaillons à partir d'exemples trouvés dans nos lectures ou sur Internet. Les exemples inventés ont été soumis à des francophones non-linguistes pour en vérifier l'interprétation.

22. Nous donnons la traduction en anglais pour illustrer le fonctionnement des deux langues. Le fonctionnement ergatif de l'anglais transpose sur le patient, le ragoût / stew, la modification qui va l'altérer. On comprend alors que la causation interne qui va commencer ne permet pas, ou difficilement, le sens métaphorique.

23. Nous suggérons que le verbe faire dans la construction causative a un opposé, laisser. Avec faire, l'agent humain qui veut modifier un certain état agit : il fait frire les pommes de terre. Avec laisser, l'agent humain ne fait rien, mais «fait » en sorte que le processus qu'il a enclenché continue à se développer. Il est intéressant de noter que dans l'énoncé fais vite frire les pommes de terre, l'adverbe temporel vite suggère au cuisinier de commencer rapidement le processus, alors qu'avec laisser, on ne peut pas avoir *laisse vite mijoter le ragoût. Mais on peut dire : laisse mijoter le ragoût pendant une heure. Et ici l'adverbe temporel porte sur le processus, mijoter. Le cuisinier doit s'abstenir d'intervenir pendant une heure. Ce qu'il fait (causation externe) est de ne rien faire.

24. Il semble difficile d'avoir : ? tu vas pourrir tes salades. L'utilisation de FAIRE introduit un agent humain dont l'action va avoir un rôle, négatif ici, sur les salades. Mais son action implique, en fait, le rôle de l'eau dans le changement d'état. La structure décompose donc le processus en sous-processus. La transitivité semble alors préférable lorsque le causateur est un élément faisant partie de l'environnement, comme l'eau, la lumière, le soleil, etc.

25. Le Trésor de la Langue Française ne donne pas d'exemple transitif, mais nous en avons trouvé plusieurs dans les blogs de cuisiniers : comment ramollir les petits biscuits arabes aux amandes? (https://www.vivelesrondes.com/forum/viewtopic_370135.htm), j'imagine que l'humidité dégagée par les congolais ramollissent les autres biscuits. (www.marmiton.org> forum > cuisine du monde). Le recours aux blogs est nécessaire pour les études de structures émergentes.

26. Nous n'avons pas discuté du concept de «causation directe » dans la mesure où l'énonciateur ne peut que postuler des relations causales entre deux événements, la plupart du temps. Pour une discussion plus complète, nous renvoyons à l'ouvrage de J. Collins, N. Hall et L.A. Paul, 2004.

\section{RÉSUMÉS}

Pour l'expression de la causation, le français, à la différence de l'anglais, n'accepte pas, pour tous les verbes, la possibilité d'être utilisé transitivement et intransitivement, et a recours à la construction en FAIRE. Ainsi, avec le verbe rebondir on peut construire la balle a rebondi, mais pas 
* Jean a rebondi la balle, et la construction avec FAIRE doit être utilisée : Jean a fait rebondir la balle. L'anglais, quant à lui, construit sans problème the ball bounced, et John bounced the ball. Deux questions se posent alors. La première question est: Est-ce que les verbes qui fonctionnent transitivement et intransitivement comme brûler, il a brûlé la lettre/la lettre a brûlé, partagent des traits sémantiques spécifiques qui expliqueraient leur rareté ? (Ben Salah Tlili (2007), travaillant à partir des travaux de J. Dubois \& F. Dubois-Charlier, Les Verbes français (1997) en dénombre 314). La deuxième question est: Faut-il prendre en considération le rôle joué par l'agent du changement d'état, et celui du patient, c'est-à-dire la distinction entre causalité intentionnelle et causalité inertielle (Guéron, 2008) ou causalité externe et causalité interne (Levin, 2009) pour comprendre les différences sémantiques que mettent en place les deux types de construction?

Unlike English, French does not freely accept the causative alternation and resorts to the FAIRE construction instead, when a verb cannot be used transitively. As opposed to John bounced the ball/ the ball bounced, we get John a fait rebondir la balle (*Jean a rebondi la balle) / la balle a rebondi. Two questions arise then. The first question is : Do the verbs which function transitively and intransitively, such as brûler (burn) : Marie a brûlé la lettre/la lettre a brûlé share semantic features that could explain their rarity? (Ben Salah Tlili (2007) drawing on J. Dubois \& F. Dubois-Charlier's dictionary, Les Verbes français (1997) suggests that there are only about 314 of them). The second question is: Do we have to take into account the role played by the agent of the change of state and the patient undergoing the change of state, namely the distinction between intentional and inertial causality (Guéron, 2008) or external and internal causation (Levin, 2009) to understand the semantic differences between the FAIRE construction and the transitive construction?

\section{INDEX}

Mots-clés : causation, externe et interne, transitivité et intransitivité, construction en FAIRE Keywords : causation, external and internal, transitivity and intransitivity, the FAIRE construction

\section{AUTEUR}

\section{GENEVIÈVE GIRARD-GILLET}

Université Sorbonne Nouvelle, PRISMES 